\title{
Care in the time of COVID-19: impact on the diagnosis and treatment of breast cancer in a large, integrated health care system
}

\author{
Annie Tang ${ }^{1} \cdot$ Elad Neeman ${ }^{2} \cdot$ Brooke Vuong $^{3} \cdot$ Vignesh A. Arasu ${ }^{4} \cdot$ Raymond Liu $^{2,5} \cdot$ Gillian E. Kuehner ${ }^{6}$. \\ Alison C. Savitz ${ }^{7}$. Liisa L. Lyon ${ }^{5}$ Prachi Anshu ${ }^{8} \cdot$ Samantha A. Seaward ${ }^{9} \cdot$ Milan D. Patel $^{10} \cdot$ Laurel A. Habel $^{5}$. \\ Lawrence H. Kushi ${ }^{5}$. Margaret Mentakis ${ }^{3}$. Eva S. Thomas ${ }^{11}$. Tatjana Kolevska ${ }^{12}$. Sharon B. Chang ${ }^{8}$ (D) on behalf of \\ The Permanente Medical Group Breast Research Collaborative
}

Received: 24 June 2021 / Accepted: 28 November 2021 / Published online: 6 January 2022

(c) The Author(s), under exclusive licence to Springer Science+Business Media, LLC, part of Springer Nature 2021

\begin{abstract}
Purposes To delineate operational changes in Kaiser Permanente Northern California breast care and evaluate the impact of these changes during the initial COVID-19 Shelter-in-Place period (SiP, 3/17/20-5/17/20).

Methods By extracting data from institutional databases and reviewing electronic medical charts, we compared clinical and treatment characteristics of breast cancer patients diagnosed 3/17/20-5/17/20 to those diagnosed 3/17/19-5/17/2019. Outcomes included time from biopsy to consultation and treatment. Comparisons were made using Chi-square or Wilcoxon rank-sum tests.

Results Fewer new breast cancers were diagnosed in 2020 during the SiP period than during a similar period in 2019 ( $n=247$ vs $n=703$ ). A higher percentage presented with symptomatic disease in 2020 than $2019(78 \%$ vs $37 \%, p<0.001)$. Higher percentages of 2020 patients presented with grade $3(37 \%$ vs $25 \%, p=0.004)$ and triple-negative tumors $(16 \%$ vs $10 \%, p=0.04)$. A smaller percentage underwent surgery first in 2020 ( $71 \%$ vs $83 \%, p<0.001)$ and a larger percentage had neoadjuvant chemotherapy ( $16 \%$ vs $11 \%, p<0.001$ ). Telehealth utilization increased from $0.8 \%$ in 2019 to $70.0 \%$ in 2020 . Times to surgery and neoadjuvant chemotherapy were shorter in 2020 than 2019 ( 19 vs 26 days, $p<0.001$, and 23 vs 28 days, $p=0.03$, respectively).

Conclusions During SiP, fewer breast cancers were diagnosed than during a similar period in 2019, and a higher proportion presented with symptomatic disease. Early-stage breast cancer diagnoses decreased, while metastatic cancer diagnoses remained similar. Telehealth increased significantly, and times to treatment were shorter in 2020 than 2019. Our system continued to provide timely breast cancer treatment despite significant pandemic-driven disruption.
\end{abstract}

Keywords COVID-19 $\cdot$ Presentation $\cdot$ Treatment times $\cdot$ Telehealth $\cdot$ Breast

Sharon B. Chang

sharon.b.chang@kp.org

1 Department of Surgery, University of California San Francisco, San Francisco, USA

2 Department of Medical Oncology, San Francisco Medical Center, Kaiser Permanente, San Francisco, USA

3 Department of Surgery, South Sacramento Medical Center, Kaiser Permanente, Sacramento, USA

4 Department of Radiology, Kaiser Permanente Vallejo Medical Center, Vallejo, USA

5 Division of Research, Kaiser Permanente Northern California, Oakland, USA

6 Department of Surgery, Kaiser Permanente Vallejo Medical Center, Vallejo, USA
7 Department of Surgery, Kaiser Permanente Walnut Creek Medical Center, Walnut Creek, USA

8 Department of Surgery, Fremont Medical Center, Kaiser Permanente, Fremont Medical Center - 39400 Paseo Padre Pkwy, Fremont, CA 94538, USA

9 Department of Radiation Oncology, Kaiser Permanente Oakland Medical Center, Oakland, USA

10 Department of Radiation Oncology, Kaiser Permanente South San Francisco Medical Center, South San Francisco, USA

11 Department of Medical Oncology, Kaiser Permanente Oakland Medical Center, Oakland, USA

12 Department of Medical Oncology, Kaiser Permanente Vallejo Medical Center, Vallejo, USA 


\section{Introduction}

The COVID-19 pandemic has disrupted all aspects of healthcare, including the diagnosis and treatment of breast cancer. In March 2020, organizations issued guidelines for cancer care to help balance the urgency of cancer treatment against the need to conserve hospital resources and minimize exposure to COVID-19 [1-3]. Recommendations included delaying breast cancer surgery when possible by initiating neoadjuvant chemotherapy (NAC) for triple-negative and HER2 + cancers and neoadjuvant endocrine therapy (NET) for some earlystage ER + HER2- cancers. However, with uncertainty about the severity and duration of the pandemic, as well as variability in facility resources and patient factors, determining which guidelines to adopt and to what extent was unclear. Several groups have described guideline-driven changes to their institutional breast care workflows during the initial phases of the pandemic [4-8], but data are limited on the clinical impact of pandemic-driven changes [9-13]. Furthermore, these reports have revealed significant variability in guideline adherence between institutions and even between physicians within the same institution [14].

Kaiser Permanente Northern California (KPNC), a large integrated health care system, charged breast cancer subspecialty directors with reviewing published guidelines, evaluating their applicability to KPNC, and recommending operational changes for breast cancer care in response to California's initial shelter-in-place (SiP) order on March 17, 2020. By May 17,2020 , the number of COVID infections had stabilized and $\mathrm{KPNC}$ resumed more normal workflows, including restarting elective surgeries which had been held since March 17. In this study, we describe the operational changes implemented during SiP (March 17-May 17, 2020), and the clinical impact of these changes on the diagnosis and treatment of breast cancer in KPNC.

\section{Methods}

KPNC is an integrated health system in Northern California serving 4.7 million patients across 21 medical centers with more than 250 outpatient facilities. This system provides both insurance coverage and healthcare to its members. KPNC membership represents the demographics of the Northern California population except at the extremes of income. This retrospective review was approved by the KPNC Institutional Review Board.

\section{Operational changes during the initial SiP period (March 17 - May 17, 2020)}

To minimize exposure to the COVID-19 virus during the initial pandemic period, KPNC leadership asked breast subspecialty leaders, most of whom are authors of this study, to develop and disseminate regional guidelines to reduce inperson visits and elective procedures for breast care (radiology, VA; surgical oncology, SBC and GK; medical oncology, $T K$ and ET; radiation oncology, MP and SS). In the Results section, we summarize the systemwide operational change guidelines we developed and disseminated.

\section{Study design, variables, and outcomes}

Table 1 summarizes the study design. We identified patients with breast cancer diagnosed March 17-May 17, 2020 and those diagnosed March 17-May 17, 2019 using the KPNC Breast Cancer Tracking System (BCTS) database, a quality assurance program that identifies incident cancers within one month of diagnosis [15]. Patients were excluded if they had a previous history of breast cancer or had been diagnosed or treated outside of KPNC. We also excluded patients who had met with a breast surgeon prior to the breast cancer diagnosis for breast symptoms such as a breast mass, or those who were referred for excisional biopsy after an image-guided core needle biopsy revealed a high-risk lesion. Finally, we excluded those who had an established medical oncologist managing another malignancy prior to the breast cancer diagnosis.

To determine patient characteristics, initial consultation type, and first treatment initiated within 5 months of diagnosis, we extracted data from the KPNC HealthConnect ${ }^{\mathrm{TM}}$ electronic health record (EHR) databases (Epic, Verona, WI, USA) and performed chart review. Data were tabulated in Excel spreadsheets. Patient characteristics included age at diagnosis, race, sex, body mass index (BMI), Charlson Comorbidity Index, presence or absence of breast-cancerrelated genetic mutations, and whether patients were diagnosed with breast cancer after screening mammography or after diagnostic imaging for breast symptoms (i.e., palpable

Table 1 Study Design

\begin{tabular}{|c|c|}
\hline Study design & Retrospective cohort \\
\hline Participants & $\begin{array}{l}\text { Patients newly diagnosed with breast cancer } \\
\text { March 17-May 17, } 2019 \text { and March 17-May } \\
\text { 17, } 2020\end{array}$ \\
\hline Data sources & $\begin{array}{l}\text { - Institutional breast cancer tracking database } \\
\text { - Electronic health records }\end{array}$ \\
\hline Data collection & $\begin{array}{l}\text { - Database queries } \\
\text { - Chart review }\end{array}$ \\
\hline Outcomes & $\begin{array}{l}\text { - Patient characteristics } \\
\text { - Tumor characteristics } \\
\text { - Consultation type, timing } \\
\text { - Treatment type, timing }\end{array}$ \\
\hline
\end{tabular}

Retrospective cohort study comparing patients newly diagnosed with breast cancer March 17, 2019 - May 17, 2019 to patients diagnosed March 17, 2020 - May 17, 2020 
breast lumps, nipple discharge, or axillary adenopathy). Tumor characteristics included histology, stage, grade, and receptor status.

Initial breast cancer consultation visits were categorized as office, telephone, or video visits for each specialty. Initial treatment categories included surgery, neoadjuvant chemotherapy (NAC), neoadjuvant endocrine therapy (NET), palliative endocrine therapy, and no treatment. We classified breast surgery as partial mastectomy or mastectomy, and nodal surgery as sentinel lymph node biopsy or axillary lymph node dissection.

Time to surgical oncology consultation was defined as the time between the biopsy date and the date of the initial surgical visit. For NAC and NET patients, time to medical oncology consultation was defined as the interval between the biopsy date and the date of the initial medical oncology visit. The time to medical oncology consultation for patients with surgery as initial treatment was defined as the time between the surgery date and the first medical oncology visit.

Time to first treatment was defined as time between the biopsy date and the date of surgery for patients who had surgery first (time to surgery, TTS), and the time between the biopsy date and the date of the first chemotherapy infusion (time to neoadjuvant chemotherapy, TTN) for patients who underwent NAC. Time to adjuvant chemotherapy was defined as time from the surgery date to the first chemotherapy infusion.

\section{Statistical analysis}

Descriptive statistics were used to examine the distributions of the demographics, clinical characteristics, and outcome variables using the mean and standard deviation (SD) or median and interquartile range (IQR) for continuous variables and frequencies and percentages for categorical variables. Comparison of demographics, types of treatment, and outcomes parameters between the 2019 and 2020 cohorts were analyzed using the chi-square or Fisher's exact test for categorical variables and Wilcoxon rank-sum tests for continuous variables. Hypothesis tests were two-sided and a $p$-value of $<0.05$ was used for statistical significance. All statistical analyses were performed using IBM SPSS $®$ Statistics (version 27.0) and SAS ${ }^{\circledR}$ SAS Institute (version 9.4).

\section{Results}

\section{Operational changes during the initial SiP period (March 17 - May 17, 2020)}

Breast Imaging: KPNC's comprehensive cancer screening and tracking program included sending patients reminders for routine cancer screening via email and paper letters, checking screening status while rooming patients for doctor appointments, and tracking each patient's completion of screening and results in a database. Before the pandemic, more than 10,000 reminders for screening mammograms were sent each week. With the SiP order on March 17, 2020, all active outreach for screening mammography was stopped, although patients wishing to have screening mammograms were accommodated. Patients referred for symptomatic breast disease and those called back from screening were evaluated with diagnostic imaging and biopsy as indicated.

Breast Cancer Diagnosis: Upon diagnosis of a breast cancer by image-guided breast biopsy, patients were referred to the facility's breast care coordinator (BCC), who conducted a telephone intake visit to answer initial questions and determine which consultations were most appropriate for each patient. To minimize the risk of exposure to COVID-19 during SiP, BCCs were encouraged to offer telehealth for initial consultations rather than office visits whenever safe and feasible.

Prospective Multidisciplinary Breast Cancer Case Conference: Every case of newly diagnosed breast cancer in KPNC was reviewed in a multidisciplinary conference before initiation of treatment. Prior to the pandemic, most of these conferences were in person, with some attendees calling in. With the SiP order, all case conferences became virtual.

Surgical Oncology Consultation and Treatment: During $\mathrm{SiP}$, initial surgery consultations occurred with a surgical oncologist only or with a multidisciplinary team consisting of a surgical oncologist, a medical oncologist, and at some facilities, a plastic surgeon and/or a radiation oncologist. Telehealth was encouraged to the extent possible for the surgeon-only appointments. Most multidisciplinary teams adopted hybrid office visit/telehealth appointments, with the patient meeting with the surgical oncologist in person, while family members and other clinical team members were present on video. Other multidisciplinary teams continued office visits with all parties present in person.

Although all KPNC elective surgeries were placed on hold due to the SiP order, cancer operations were not considered elective and were encouraged to continue. Surgical oncology directors developed guidelines during $\mathrm{SiP}$ about the timing and extent of surgical treatment based on patient factors, tumor characteristics, and the ability of the medical center to continue breast cancer surgeries safely (Table 2). Healthy patients with operable invasive tumors were encouraged to undergo surgery, especially if they had aggressive tumor subtypes and/or nodal disease. Patients with ductal carcinoma in situ (DCIS) were offered the option of waiting. However, final treatment decisions were left to the discretion of individual surgical oncologists and patients.

Medical Oncology Consultation and Treatment: Neoadjuvant endocrine therapy (NET) was offered to ER + patients 
for whom the risks of surgery during SIP were felt to outweigh the benefits due to the patients' medical comorbidities and relatively indolent tumors (Table 2). Patients who met criteria for NAC but had operable tumors were encouraged to undergo surgery first. Adjuvant chemotherapy for $\mathrm{HR}+1$ HER2- was considered for those with node-positive, or borderline/high Oncotype Dx scores. The use of Oncotype Dx to guide chemotherapy recommendations for those with 1-3 positive nodes was also considered, even before RxPonder trial results were presented in December 2020 [16]. Finally, starting adjuvant endocrine therapy while awaiting radiation treatment was encouraged if a delay in starting radiation was anticipated.

Endocrine therapy alone was considered for those with metastatic ER +/HER2- disease. For HER2 + metastatic disease, HER2 antibody treatment was considered, and endocrine therapy was recommended for ER +/HER2 + patients. For triple-negative metastatic disease, chemotherapy was initiated for some patients after weighing the risks and benefits.

Intervals for routine port flushing were increased from 1 to 3 months to reduce unnecessary visits, and elective infusions were postponed. However, patients who wished to continue elective infusions were accommodated.

\section{Presentation and diagnosis}

A total of 1,681 screening mammograms were performed March 17-May 17, 2020, in contrast to the 180,724 mammograms performed March 17-May 17, 2019. During the 2 month period, only 54 breast cancers were detected by screening in the 2020 cohort, compared to 440 in the 2019 group.

Table 3 shows the demographic and clinical characteristics of the 703 patients in the 2019 cohort and the 247 patients in the 2020 cohort. The cohorts did not differ significantly by race, BMI, comorbidities, genetic mutation status, node positivity, or histologic subtypes. A higher percentage of patients were $<65$ years in the 2020 cohort $(66 \%$ vs $57 \%$ in $2019, p=0.04$ ). While the total number of breast cancer diagnoses was smaller in the 2020 cohort, a higher percentage of patients presented with symptomatic disease in 2020 than in 2019 , $\left(78 \%\right.$ vs $37 \%, X^{2}(1, n=950)=121.5$, $p<0.001)$. T-stages at presentation were higher in 2020 : $78 \%$ of patients presented with T1c or greater tumors in 2020 vs $64 \%$ in $2019\left(X^{2}(1, n=950),=15.1, p<0.001\right)$. Absolute numbers of patients presenting with metastatic disease were similar in 2020 and 2019 (18 in 2020 and 17 in 2019), although with fewer diagnoses in 2020, these patients comprised a higher percentage of the cohort (7\% in $2020 \mathrm{vs}$ $2 \%$ in $2019, p=0.001$ ). Of patients with invasive disease, a higher percentage presented with grade 3 tumors in 2020
(37\% in 2020 vs $25 \%$ in $2019, p=0.004$ ), and triple-negative tumors $(16 \%$ vs $10 \%, p=0.04)$.

\section{Initial consultation timing and types}

The total number of surgical oncology consultations decreased from 533 in 2019 to 169 in 2020 (Table 4). Medical oncology consultations for neoadjuvant therapy decreased from 94 in 2019 to 63 in 2020, and adjuvant therapy decreased from 182 to 78 . Time to initial consultation was shorter in 2020 than 2019 for both surgical oncology consultations (median 7 vs 11 days, $p<0.001$ ) and medical oncology consultations after surgery (median 13 vs 15 days, $p=0.01$ ), but did not differ for neoadjuvant medical oncology consultations (median 12 vs 13.5 days, $p=0.19$ ). Telehealth utilization for all initial consultations was higher in 2020 than 2019 (70.0\% vs $0.8 \%, p<0.001)$. For patients diagnosed in 2020 , there were no significant differences in proportion of initial telehealth vs office consultation visits for patients $\mathrm{BMI}<30$ and $\mathrm{BMI} \geq 30\left(\mathrm{X}^{2}(1, n=238)=1.86\right.$, 0.17 and $\mathrm{X}^{2}(1, n=241)=0.33, p=0.56$, respectively $)$. Telehealth comprised $58 \%$ of initial surgical oncology consultations in 2020 , compared to $0 \%$ in 2019. Similarly, a significantly higher percentage of initial medical oncology consultations in 2020 were telehealth than in 2019 (85.1\% vs $2.5 \%, p<0.001)$.

Time to initial surgical consultation for telehealth appointments in 2020 was significantly shorter than for office visits (median 7 days vs 9 days, $p=0.03$ ) (Table 4). Time to initial medical oncology consultation for patients with NAC/NET was not significantly different between visit types (median 12 days for telehealth vs 9.5 days for office visits, $p=0.60$ ). Similarly, we found no difference in time to medical oncology consultation by visit type for patients with adjuvant chemotherapy (median 13 days for telehealth vs 13 days for office visits, $p=0.82$ ).

\section{Initial treatment types and intervals}

Although most patients underwent surgery as their first treatment in 2020, the percentage was smaller (71\% in 2020 vs $83 \%$ in $2019, p<0.001)$, and a larger percentage started NAC than in 2019 ( $16 \%$ in 2020 vs $11 \%$ in $2019, p<0.001)$ (Table 5). Six percent $(n=14)$ of the overall 2020 cohort received NET while awaiting surgery. However, this represented $46.7 \%$ of NET candidate patients ( $\geq 60$ years with T1, HR +/HER2-, grade 1 or 2 breast cancer; $n=30$ ). A higher proportion of patients underwent mastectomy in 2020 than in 2019 (33\% vs $24 \%, p=0.02$ ).

Time to surgery (TTS) was significantly shorter for the 2020 group (median 19 days vs 26 days in $2019, p<0.001$ ) (Table 5). Time to neoadjuvant chemotherapy (TTN) also 


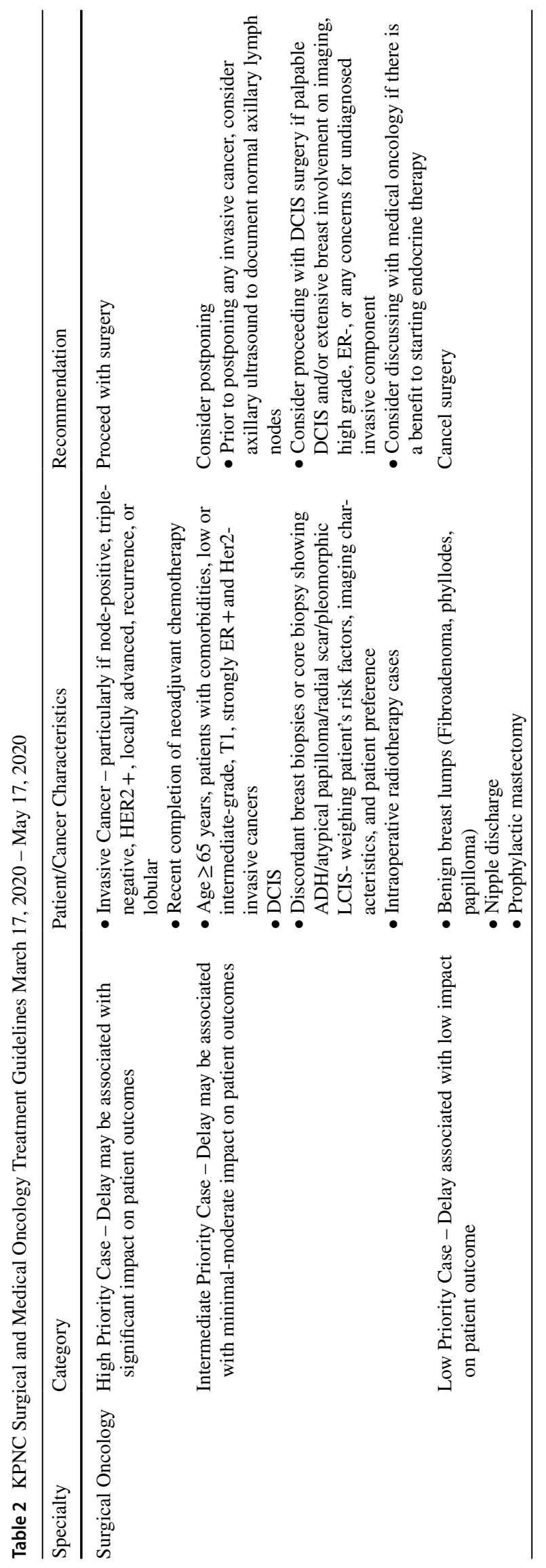




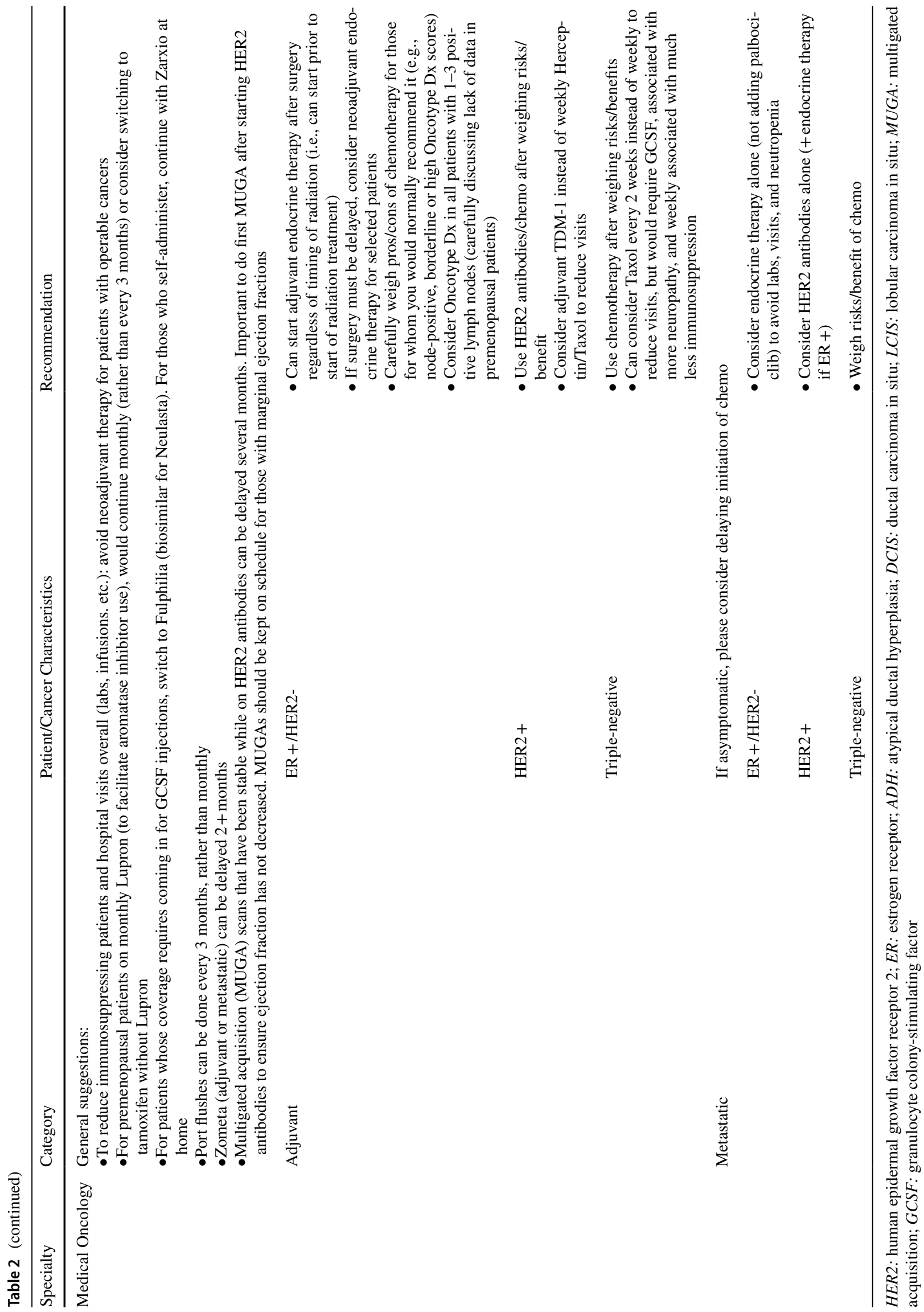


Table 3 Demographic and Clinical Characteristics of Patients Diagnosed with Breast Cancer March 17 - May 17, 2019 vs. March 17 - May 17, 2020

\begin{tabular}{|c|c|c|c|}
\hline Characteristic & $2019(n=703)$ & $2020(n=247)$ & \\
\hline & No $(\%)$ & No $(\%)$ & $p$-value \\
\hline Age, years & & & 0.04 \\
\hline$<40$ & $25(4)$ & $14(6)$ & \\
\hline $40-64$ & $373(53)$ & $147(60)$ & \\
\hline$\geq 65$ & $305(43)$ & $86(35)$ & \\
\hline Race & & & 0.37 \\
\hline White & $386(55)$ & $143(58)$ & \\
\hline Asian & $143(20)$ & $41(17)$ & \\
\hline Hispanic & $100(14)$ & $29(12)$ & \\
\hline Black & $44(6)$ & $19(8)$ & \\
\hline Other/Unknown & $30(4)$ & $15(6)$ & \\
\hline BMI, $N(\%)$ & & & 0.63 \\
\hline Obese $(\mathrm{BMI} \geq 30)$ & $268(38)$ & $90(36)$ & \\
\hline $\begin{array}{l}\text { Charlson Comorbidity } \\
\text { Index }\end{array}$ & & & 0.73 \\
\hline Greater than 3 & $88(12)$ & $33(13)$ & \\
\hline Genetic Mutation & $24(3)$ & $15(6)$ & 0.07 \\
\hline Detection Method & & & $<0.001$ \\
\hline Screening Mammogram & $440(63)$ & $54(22)$ & \\
\hline Symptomatic & $263(37)$ & $193(78)$ & \\
\hline Histology & & & 0.16 \\
\hline DCIS & $95(14)$ & $21(9)$ & \\
\hline IDC & 489 (70) & $184(75)$ & \\
\hline ILC & $64(9)$ & $26(11)$ & \\
\hline Other & $55(8)$ & $16(7)$ & \\
\hline T Stage & & & 0.02 \\
\hline Tis & $95(14)$ & $21(9)$ & \\
\hline $\mathrm{T} 1 \mathrm{mi}$ & $16(2)$ & $6(2)$ & \\
\hline T1a & $46(7)$ & $7(3)$ & \\
\hline $\mathrm{T} 1 \mathrm{~b}$ & $90(13)$ & $19(8)$ & \\
\hline $\mathrm{T} 1 \mathrm{c}$ & 177 (25) & $66(27)$ & \\
\hline $\mathrm{T} 2$ & $217(31)$ & $98(40)$ & \\
\hline $\mathrm{T} 3$ & $37(5)$ & $18(7)$ & \\
\hline $\mathrm{T} 4$ & $21(3)$ & $10(4)$ & \\
\hline $\mathrm{Tx} / \mathrm{T} 0$ & $4(0)$ & $2(1)$ & \\
\hline$\geq \mathrm{T} 1 \mathrm{c}$ & $452(64)$ & $192(78)$ & $<0.001$ \\
\hline N Stage $^{\mathrm{b}}$ & & & 0.88 \\
\hline N1 & $125(69)$ & $60(68)$ & \\
\hline $\mathrm{N} 1 \mathrm{~m}$ & $20(11)$ & $8(9)$ & \\
\hline $\mathrm{N} 2$ & $14(8)$ & $9(10)$ & \\
\hline N3 & $21(12)$ & $11(13)$ & \\
\hline M Stage & & & 0.001 \\
\hline M0 & $685(97)$ & $227(92)$ & \\
\hline M1 & $17(2)$ & $18(7)$ & \\
\hline Mx & $1(0)$ & $2(1)$ & \\
\hline Invasive Cancer & $608(87)$ & $229(91)$ & 0.03 \\
\hline Grade & & & 0.004 \\
\hline 1 & $166(27)$ & $44(20)$ & \\
\hline
\end{tabular}

Table 3 (continued)

\begin{tabular}{llll}
\hline Characteristic & $2019(n=703)$ & $2020(n=247)$ & \\
\hline 2 & $277(46)$ & $95(42)$ & \\
3 & $149(25)$ & $83(37)$ & \\
Unknown & $16(3)$ & $4(2)$ & 0.04 \\
Receptor Status & & & \\
HR +/HER2- & $460(76)$ & $150(67)$ & \\
HR +/HER2+ & $55(9)$ & $29(13)$ & \\
HR-/HER2 + & $28(5)$ & $9(4)$ \\
HR-/HER2- & $59(10)$ & $35(16)$ \\
Unknown & $4(1)$ & $2(1)$ \\
\hline
\end{tabular}

BMI: Body Mass Index; DCIS: Ductal Carcinoma In Situ; IDC: Invasive Ductal Carcinoma; ILC: Invasive Lobular Carcinoma; HR: Hormone Receptor; HER2: Herceptin Epidermal Growth Factor Receptor

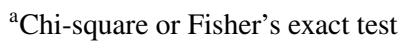

${ }^{\mathrm{b}}$ Total $n=268$

was shorter for the 2020 group (median 23 days vs 28 days in $2019, p=0.03)$. Time to adjuvant chemotherapy did not differ between 2020 and 2019 (34 days vs 37 days, $p=0.09$ ).

\section{Discussion}

We describe operational changes in multidisciplinary breast cancer management and the diagnostic and treatment impact of these changes within a large, integrated health care system during SiP (Fig. 1). We found that screening mammography rates fell when screening mammography outreach was stopped. As a likely consequence, significantly fewer patients were diagnosed with breast cancer in 2020 than in 2019 , and a larger proportion presented with symptomatic disease. The promotion of telehealth during SiP increased telehealth utilization significantly and probably contributed to decreased times to initial consultation. Finally, TTS and TTN were significantly shorter in 2020 . We hypothesize this was due to increased operating room and infusion chair availability secondary to postponement of elective procedures and interventions. To our knowledge, our study is the first to describe both the systemwide operational changes and the likely sequelae of these changes on breast cancer management in an integrated health care system.

Others have described operational changes in their management of breast cancer [4-7, 11, 17]. One group promoted the use of neoadjuvant systemic therapies to delay definitive surgery until it had personal protective equipment and resources to resume breast cancer surgery [18]. KPNC breast cancer directors had considered using systemic therapy to temporize the need for surgery, but recommended against NAC in surgical candidates because of the high mortality risk in chemotherapy patients who contracted a COVID 
Table 4 Initial New Physician Consultation Types and Timing by Specialty, for Patients Diagnosed with Breast Cancer March 17 - May 17, 2019 vs. March 17 - May 17, 2020

\begin{tabular}{|c|c|c|c|c|c|c|}
\hline \multirow[t]{2}{*}{ Type of Visit } & \multicolumn{2}{|c|}{ Total Visits, No. (\%) } & \multirow[t]{2}{*}{$p$-value ${ }^{\mathrm{a}}$} & \multicolumn{2}{|c|}{ Median Days to Consultation (IQR) } & \multirow[t]{2}{*}{$p$-value } \\
\hline & 2019 & 2020 & & 2019 & 2020 & \\
\hline All Patients & & & $<0.001$ & & & N/A \\
\hline Office Visits & $822(99.2 \%)$ & $92(29.6 \%)$ & & N/A & N/A & \\
\hline Telehealth Visits & $7(0.8 \%)$ & $219(70.4 \%)$ & & N/A & N/A & \\
\hline Video Visits & $1(0.1 \%)$ & $130(41.8 \%)$ & & N/A & N/A & \\
\hline Telephone Visits & $6(0.7 \%)$ & $88(28.3)$ & & N/A & N/A & \\
\hline Surgical Oncology First ${ }^{\mathrm{b}}$ & & & $<0.001$ & $11(8-13)$ & $7(6-11)$ & $<0.001$ \\
\hline Office Visits & $553(100 \%)$ & $71(42.0 \%)$ & & $11(8-13)$ & $9(7-12)$ & 0.12 \\
\hline Telehealth Visits & $0(0 \%)$ & $98(58.0 \%)$ & & N/A & $7(5-9.25)$ & N/A \\
\hline Video Visits & $0(0 \%)$ & $57(33.7 \%)$ & & N/A & $7(5.5-10)$ & N/A \\
\hline Telephone Visits & $0(0 \%)$ & $41(24.3 \%)$ & & N/A & $7(4-9)$ & N/A \\
\hline All Medical Oncology ${ }^{c}$ & & & $<0.001$ & & & N/A \\
\hline Office Visits & $269(97.5 \%)$ & $21(14.9 \%)$ & & N/A & N/A & \\
\hline Telehealth Visits & $7(2.5 \%)$ & $120(85.1 \%)$ & & N/A & N/A & \\
\hline Video Visits & $1(0.4 \%)$ & $73(51.8 \%)$ & & N/A & N/A & \\
\hline Telephone Visits & $6(2.2 \%)$ & $47(33.3 \%)$ & & N/A & N/A & \\
\hline Neoadjuvant Medical Oncology & & & $<0.001$ & $13.5(10.5-21)$ & $12(7-15)$ & 0.19 \\
\hline Office Visits & $93(98.9 \%)$ & $18(28.6 \%)$ & & $14(11-21)$ & $9.5(6.75-14.0)$ & 0.28 \\
\hline Telehealth Visits & $1(1 \%)$ & $45(71.4 \%)$ & & N/A & $12(8-17)$ & N/A \\
\hline Video Visits & $1(1 \%)$ & $23(35.9 \%)$ & & N/A & $10(7-13)$ & N/A \\
\hline Telephone Visits & $0(0 \%)$ & $22(34.4 \%)$ & & N/A & $15(10-20.5)$ & N/A \\
\hline Adjuvant Medical Oncology ${ }^{\mathrm{e}}$ & & & $<0.001$ & $15(10-21)$ & $13(8-19)$ & 0.01 \\
\hline Office Visits & $176(96.7 \%)$ & $3(3.8 \%)$ & & $15(10.25-21)$ & $13(13-16)$ & 0.95 \\
\hline Telehealth Visits & $6(3.3 \%)$ & $75(96.2 \%)$ & & $12.5(7.75-21.5)$ & $13(8-19)$ & 0.89 \\
\hline Video Visits & $0(0 \%)$ & $50(64.1 \%)$ & & N/A & $14(9-21)$ & N/A \\
\hline Telephone Visits & $6(3.3 \%)$ & $25(32.1 \%)$ & & $12.5(7.75-21.5)$ & $11(7-14)$ & 0.71 \\
\hline
\end{tabular}

N/A: not applicable

${ }^{a}$ Chi-square or Fisher's exact test for categorical variables, Wilcoxon rank-sum test for continuous variables

${ }^{b}$ Patients who had surgery first including those who had adjuvant chemotherapy and excluding those who were previously followed by surgery prior to cancer diagnosis, Total $n=722$

${ }^{\mathrm{c}}$ Total $=417$

${ }^{\mathrm{d}}$ Patients who had either chemotherapy or endocrine therapy first, Total $n=157$

${ }^{\mathrm{e}}$ Total $n=261$

infection [19]. The "surgery first" approach was possible at KPNC because our facilities had the capacity to continue cancer operations during the initial SiP period, likely resulting in significantly shorter TTS in the 2020 cohort compared to the 2019 cohort. In contrast, others have reported delays in breast cancer treatment during the pandemic [11, 12, 20, 21]. A multicenter review of 432 patients revealed delays in breast cancer treatment during the initial period of the COVID-19 pandemic compared to pre-pandemic treatment times [12], and in a survey of patients with breast cancer, $44 \%$ reported delays in their cancer treatment [20].

The number of new breast cancer diagnoses decreased by $65 \%$, similar to the decreases of $52-67 \%$ described by others in association with decreased outreach and screening mammography during $\operatorname{SiP}[9,10,13]$. We hypothesize that the higher proportions of symptomatic disease and more aggressive tumors in 2020 than in 2019 reflect the changes in screening mammography outreach protocols rather than disease progression within the two-month initial SiP period. Despite the higher percentage of patients presenting with symptomatic disease, we noted a $27 \%$ decrease in the absolute number of symptomatic patients diagnosed, suggesting that many symptomatic patients may not have sought evaluation during SiP. An estimated $41 \%$ of U.S. adults reported delaying medical care during the pandemic due to concerns about COVID-19, and up to $12 \%$ avoided urgent or emergency care because of these concerns [22]. COVID-19 anxiety and fear in cancer patients has been associated with 
Table 5 First Treatment Type and Treatment Timing for Patients Diagnosed with Breast Cancer March 17 - May 17, 2019 vs. March 17 - May 17, 2020

Fig. 1 Summary of KPNC Breast Care Operational Changes During the Initial SiP Period (March 17, 2020 - May 17, 2020) and Clinical Impact. Abbreviations: KPNC: Kaiser Permanente Northern California; SiP: Shelter in Place

\begin{tabular}{|c|c|c|c|}
\hline & $2019(n=703)$ & $2020(n=247)$ & $p$-value $^{\mathrm{a}}$ \\
\hline & No. $(\%)$ & No. $(\%)$ & \\
\hline First treatment type & & & $<0.001$ \\
\hline Surgery & $583(83)$ & $176(71)$ & \\
\hline Neoadjuvant Chemotherapy & $75(11)$ & $40(16)$ & \\
\hline Neoadjuvant HT & $3(0)$ & $14(6)$ & \\
\hline Palliative Endocrine & $20(3)$ & $10(4)$ & \\
\hline No treatment & $22(3)$ & $7(3)$ & \\
\hline Breast operations ${ }^{\mathrm{b}}$ & & & 0.02 \\
\hline Partial Mastectomy & $441(76)$ & $117(67)$ & \\
\hline Mastectomy & $139(24)$ & $57(33)$ & \\
\hline Lymph node operations $^{c}$ & & & 0.69 \\
\hline ALND & $123(23)$ & $39(24)$ & \\
\hline SLNB & $422(77)$ & $123(76)$ & \\
\hline \multicolumn{4}{|l|}{ First treatment timing } \\
\hline Time to Surgery ${ }^{\mathrm{d}}$ : median days (IQR) & $26(20-36)$ & $19(14-27)$ & $<0.001$ \\
\hline Time to Neoadjuvant Chemotherapye: median days (IQR) & $28(21-32)$ & $23(18-28)$ & 0.03 \\
\hline Time to Adjuvant Chemotherapy ${ }^{\mathrm{f}}$ : median days (IQR) & $37(30-48)$ & $34(29-44)$ & 0.10 \\
\hline
\end{tabular}

HT: Hormone therapy; ALND: Axillary Lymph Node Dissection; SLNB: Sentinel Lymph Node Biopsy; $I Q R$ : Interquartile range

${ }^{\mathrm{a}}$ Chi-square or Wilcoxon rank-sum tests

${ }^{\mathrm{b}}$ Total $n=754 ;{ }^{\mathrm{c}}$ Total $n=707$

${ }^{\mathrm{d}}$ Total $n=759$

${ }^{\mathrm{e}}$ Total $n=103$

${ }^{\mathrm{f}}$ Total $n=261$

\section{Operational Change Consequence Clinical Impact}

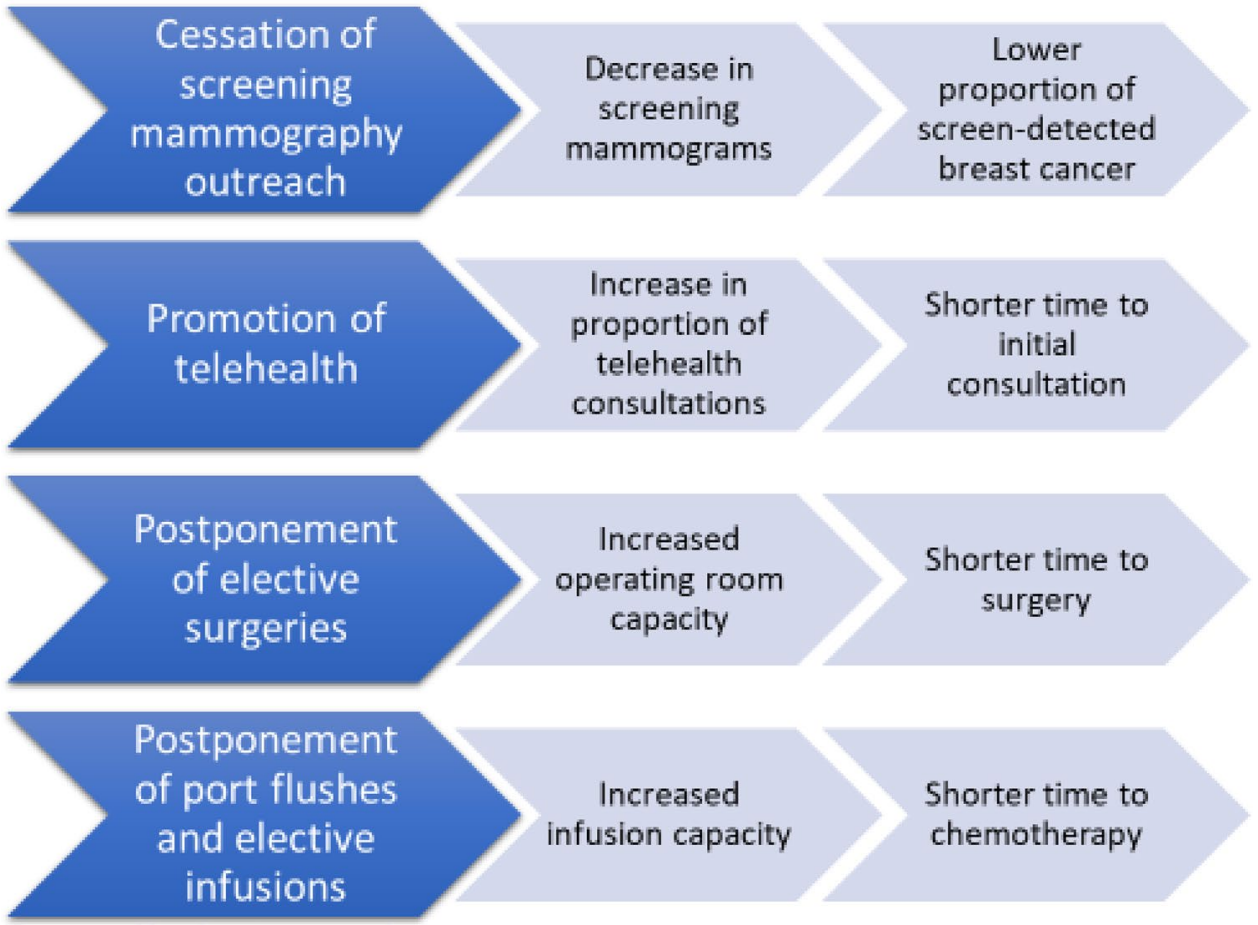


treatment delays and treatment refusal [12, 23]. Our results highlight the potential for disease progression as patients continue to delay care during the pandemic.

The significant decreases in TTS and TTN were unexpected. With national organizations recommending postponement of surgery for patients with breast cancer [2] and patients' reluctance to come to the hospital for fear of the risk of COVID infection [24], we expected average TTS to be longer in the pandemic cohort. However, continuation of cancer operations in KPNC was encouraged as long as facilities could perform them safely. We presume that the increased operating room capacity due to postponement of elective operations, combined with the smaller volume of breast cancer patients, contributed to the shorter times to surgery. Telehealth also may have contributed to shorter TTS, allowing shorter times to initial consultation. Similarly, the shorter TTN likely was due to the increased availability of chemotherapy infusion chairs, since routine port flushes and elective infusions were postponed.

Telehealth utilization increased significantly across specialties for initial breast cancer consultations during SiP, with telehealth visits comprising $58 \%$ of initial surgical consultations in the 2020 cohort. This is consistent with the increase in telehealth medical oncology consultations reported for all cancer types in TPMG [25]. Others also have described a significant increase in cancer care video visits [26] and telehealth outpatient surgical consultations during SiP [27]. Cancer patients may prefer telehealth in order to minimize the number of medical facility visits and the risk of contracting COVID-19 [24]. Telehealth utilization may decrease as the pandemic is contained, but we anticipate it will become an established part of many practices now that both patients and providers have experienced its convenience and ease.

Our study has several limitations. First, we did not capture clinical outcomes. Due to the relative recentness of the pandemic, the impact of treatment algorithm changes on longterm outcomes such as local recurrence and breast cancer specific mortality may not become evident until years in the future. Comparisons of shorter-term outcomes such as emergency department (ED) visits and readmissions most likely were confounded significantly by pandemic-driven changes in ED workflows, hospital admission criteria, and patient reluctance to seek medical care. With these confounding factors and our historically low rate of complications [28, 29], we felt it was unlikely that we could draw meaningful conclusions regarding such shorter-term outcomes. A second limitation is that comparisons between cohorts from the two time periods were not adjusted for potential confounders. Finally, with the retrospective nature of our study, we did not capture patient-reported outcomes to evaluate patient satisfaction with telehealth, NET, and other care delivery changes driven by the pandemic.
In conclusion, our study describes operational changes and their clinical impact on breast cancer management in KPNC during the initial SiP period. Our study highlights the importance of screening mammography in the early detection of breast cancer, and we recommend that screening mammography be continued whenever safe and feasible to minimize the number of undetected cancers. We also demonstrate the efficacy of telehealth in cancer care and its value when inperson visits are not safe or feasible. Finally, we describe the capacity of an integrated health system to continue providing timely breast cancer treatment despite the constraints of the pandemic. Rapid implementation of coordinated workflow changes enabled continuation of breast cancer care even in the face of great uncertainty and resource limitations.

Looking forward, we are concerned about the prognosis of patients in our system with undiagnosed cancer. Although screening mammography outreach has resumed, as of December 8, 2020 screening mammography rates still were less than $50 \%$ of pre-pandemic levels because patients declined screening mammography. Given the $65 \%$ decrease in overall breast cancer diagnoses but the stable number of stage IV diagnoses, we suspect that the majority of undiagnosed patients have stage $1-3$ disease, and we are concerned that stage progression could occur due to pandemic-related delays in diagnosis. Further studies on the long-term consequences of delay in breast cancer screening and diagnosis are warranted. We anticipate that with COVID vaccinations and better control of the pandemic, more patients will proceed with screening. In the meantime, breast care programs must prepare for a likely surge of new breast cancer patients in the near future.

Acknowledgements The authors would like to thank Pamela Derish in the UCSF Department of Surgery for assistance in editing this manuscript and Jane Bethard-Tracy from the KPNC Breast Cancer Tracking Service for assistance with data collection.

Funding Funding for this study was provided by Kaiser Permanente Northern California Graduate Medical Education, Kaiser Foundation Hospitals. Kaiser permanente

Data availability The datasets generated during and/or analyzed during the current study are not publicly available due to patient privacy laws, but are available from the corresponding author upon reasonable request.

Code availability Not applicable.

\section{Declarations}

Conflict of interest The authors report no conflicts of interest in any product mentioned or concept discussed in this article. This study has not been presented or published. An oral presentation with parts of this study was given at the 2020 San Antonio Breast Cancer Symposium on December 8, 2020. The authors have not published, posted, or submitted any related papers from the same study for publication. 


\section{References}

1. Dietz JR, Moran MS, Isakoff SJ et al (2020) Recommendations for prioritization, treatment, and triage of breast cancer patients during the COVID-19 pandemic. The COVID-19 pandemic breast cancer consortium. Breast Cancer Res Treat 181:487-497. https:// doi.org/10.1007/s10549-020-05644-z

2. Bartlett DL, Howe JR, Chang G et al (2020) Management of cancer surgery cases during the COVID-19 pandemic: considerations. Ann Surg Oncol 27:1717-1720. https://doi.org/10.1245/ s10434-020-08461-2

3. COVIDSurg Collaborative, (2020) Global guidance for surgical care during the COVID-19 pandemic. Br J Surg 107:1097-1103. https://doi.org/10.1002/bjs.11646

4. Al-Shamsi HO, Alhazzani W, Alhuraiji A et al (2020) A practical approach to the management of cancer patients during the novel coronavirus disease 2019 (COVID-19) pandemic: an international collaborative group. Oncologist 25:e936-e945. https://doi.org/10. 1634/theoncologist.2020-0213

5. Soran A, Gimbel M, Diego E (2020) Breast cancer diagnosis, treatment and follow-up during COVID-19 pandemic. Eur J Breast Health 16:86-88. https://doi.org/10.5152/ejbh.2020.240320

6. Chan JJ, Sim Y, Ow SGW et al (2020) The impact of COVID-19 on and recommendations for breast cancer care: the Singapore experience. Endocr Relat Cancer 27:R307-R327. https://doi.org/ 10.1530/ERC-20-0157

7. Ribeiro R, Wainstein AJA, de Castro Ribeiro HS et al (2021) Perioperative cancer care in the context of limited resources during the COVID-19 pandemic: Brazilian society of surgical oncology recommendations. Ann Surg Oncol 28:1289-1297. https:// doi.org/10.1245/s10434-020-09098-x

8. Zarzaur BL, Stahl CC, Greenberg JA et al (2020) Blueprint for restructuring a department of surgery in concert with the health care system during a pandemic: the university of Wisconsin experience. JAMA Surg 155:628. https://doi.org/10.1001/jamasurg. 2020.1386

9. Kaufman HW, Chen Z, Niles J, Fesko Y (2020) Changes in the number of us patients with newly identified cancer before and during the coronavirus disease 2019 (COVID-19) pandemic. JAMA Netw Open 3:e2017267. https://doi.org/10.1001/jamanetwor kopen.2020.17267

10. Nyante SJ, Benefield TS, Kuzmiak CM, Population-level impact of coronavirus disease, et al (2019) On breast cancer screening and diagnostic procedures. Cancer n/a: https://doi.org/10.1002/ cncr.33460

11. Hawrot K, Shulman LN, Bleiweiss IJ, et al (2021) Time to Treatment Initiation for Breast Cancer During the 2020 COVID-19 Pandemic. JCO Oncology Practice OP.20.00807. https://doi.org/ 10.1200/OP.20.00807

12. Vanni G, Tazzioli G, Pellicciaro M, et al (2020) Delay in Breast Cancer Treatments During the First COVID-19 Lockdown A Multicentric Analysis of 432 Patients. Anticancer Res 40:7119-7125 Doi: https://doi.org/10.21873/anticanres.14741

13. Li J, Wang H, Geng C et al (2020) Suboptimal declines and delays in early breast cancer treatment after COVID-19 quarantine restrictions in China: a national survey of 8397 patients in the first quarter of 2020. EClinicalMedicine 26:100503. https://doi.org/10. 1016/j.eclinm.2020.100503

14. Mallick I, Chakraborty S, Baral S et al (2021) Prioritizing delivery of cancer treatment during a COVID-19 lockdown: the experience of a clinical oncology service in India. JCO Glob Oncol 7:99-107. https://doi.org/10.1200/GO.20.00433

15. Callahan M, Sanderson J (2000) A breast cancer tracking system. Perm J 4:36-39
16. Kalinsky K, Barlow WE, Meric-Bernstam F, et al (2021) Abstract GS3-00: First results from a phase III randomized clinical trial of standard adjuvant endocrine therapy $(\mathrm{ET})+/$ - chemotherapy (CT) in patients (pts) with 1-3 positive nodes, hormone receptor-positive (HR+) and HER2-negative (HER2-) breast cancer (BC) with recurrence score $(\mathrm{RS})<25$ : SWOG S1007 (RxPonder). Cancer Res 81:GS3-GS3-00. https://doi.org/10.1158/1538-7445.SABCS 20-GS3-00

17. Satish T, Raghunathan R, Prigoff JG, et al (2021) Care Delivery Impact of the COVID-19 Pandemic on Breast Cancer Care. JCO Oncology Practice OP.20.01062. https://doi.org/10.1200/OP.20. 01062

18. Sheng JY, Santa-Maria CA, Mangini N et al (2020) Management of breast cancer during the COVID-19 pandemic: a stage- and subtype-specific approach. JCO Oncol Pract 16:665-674. https:// doi.org/10.1200/OP.20.00364

19. Liang W, Guan W, Chen R et al (2020) Cancer patients in SARSCoV-2 infection: a nationwide analysis in China. Lancet Oncol 21:335-337. https://doi.org/10.1016/S1470-2045(20)30096-6

20. Papautsky EL, Hamlish T (2020) Patient-reported treatment delays in breast cancer care during the COVID-19 pandemic. Breast Cancer Res Treat 184:249-254. https://doi.org/10.1007/ s10549-020-05828-7

21. Wu J, Bobo S, Henry S, et al (2021) Abstract PS6-32: Impact of COVID-19 on breast cancer care at a Bay Area academic center. Cancer Res 81:PS6-PS6-32. https://doi.org/10.1158/1538-7445. SABCS20-PS6-32

22. Czeisler MÉ, Marynak K, Clarke KEN, et al (2020) Delay or Avoidance of Medical Care Because of COVID-19-Related Concerns - United States, June 2020. MMWR Morb Mortal Wkly Rep 69:1250-1257. https://doi.org/10.15585/mmwr.mm6936a4

23. Guven DC, Sahin TK, Aktepe OH et al (2020) Perspectives, Knowledge, and fears of cancer patients about COVID-19. Front Oncol 10:1553. https://doi.org/10.3389/fonc.2020.01553

24. Vanni G, Materazzo M, Pellicciaro M, et al (2020) Breast Cancer and COVID-19: The Effect of Fear on Patients' Decision-making Process. In Vivo 34:1651-1659. https://doi.org/10.21873/invivo. 11957

25. Liu R, Sundaresan T, Reed ME et al (2020) Telehealth in oncology during the COVID-19 outbreak: bringing the house call back virtually. JCO Oncology Practice 16:289-293. https://doi.org/10. 1200/OP.20.00199

26. Lonergan PE, Washington Iii SL, Branagan L et al (2020) Rapid Utilization of telehealth in a comprehensive cancer center as a response to COVID-19: cross-sectional analysis. J Med Internet Res 22:e19322. https://doi.org/10.2196/19322

27. Lazar AN, Nemeth SK, Kurlansky PA et al (2021) Adoption and usage of video telehealth in a large, academic department of surgery. Annals of Surgery Open 2:e040. https://doi.org/10.1097/ AS9.0000000000000040

28. Vuong B, Dusendang JR, Chang SB et al (2021) Outpatient mastectomy: factors influencing patient selection and predictors of return to care. J Am Coll Surg 232:35-44. https://doi.org/10. 1016/j.jamcollsurg.2020.09.015

29. Patel AR, Vuong B, Kuehner GE et al (2020) Adoption of opioidsparing and non-opioid regimens after breast surgery in a large, integrated health care delivery system. Ann Surg Oncol 27:48354843. https://doi.org/10.1245/s10434-020-08897-6

Publisher's Note Springer Nature remains neutral with regard to jurisdictional claims in published maps and institutional affiliations. 